\title{
Bayesian Analysis of the Weibull Paired Comparison Model Using Numerical Approximation
}

\author{
Khalil Ullah $\mathbb{D}^{1,2}$ and Muhammad Aslam $\mathbb{D}^{2}$ \\ ${ }^{1}$ Department of Sciences and Humanities, National University of Computer and Emerging Sciences, Islamabad, Pakistan \\ ${ }^{2}$ Department of Mathematics and Statistics, Riphah International University, Islamabad, Pakistan \\ Correspondence should be addressed to Khalil Ullah; khalil.awan@nu.edu.pk
}

Received 9 October 2020; Revised 12 November 2020; Accepted 3 December 2020; Published 15 December 2020

Academic Editor: Hijaz Ahmad

Copyright (c) 2020 Khalil Ullah and Muhammad Aslam. This is an open access article distributed under the Creative Commons Attribution License, which permits unrestricted use, distribution, and reproduction in any medium, provided the original work is properly cited.

\begin{abstract}
The method of paired comparisons (PC) is widely used to rank items using sensory evaluations. The PC models are developed to provide basis for such comparisons. In this study, the Weibull PC model is analyzed under the Bayesian paradigm using noninformative priors and different loss functions, namely, Squared Error Loss Function (SELF), Quadratic Loss Function (QLF), DeGroot Loss Function (DLF), and Precautionary Loss Function (PLF). Numerical approximation is used to illustrate the entire estimation procedure. A real dataset showing usage preferences for different cellphone brands, Huawei (HW), Samsung (SS), Oppo (OP), QMobile (QM), and Nokia (NK), is used. Quadrature method is used to evaluate the Bayes estimates, their posterior risks, preference probabilities, predictive probabilities, and posterior probabilities to establish and verify ranking order of the competing cellphone brands under study. The results show that the paired comparison model under the study using Bayesian approach involving various loss functions can offer mathematical approach to evaluate cellphone brand preferences. The ranking provided by the model is justifiable according to the usage preference for these cellphone brands. The ranking given by the model indicates that cellphone brand Samsung is preferred the most and QMobile is the least preferred. The plausibility of the model is also assessed using the Chi square test of goodness of fit.
\end{abstract}

\section{Introduction}

Paired Comparison (PC) is a very helpful technique for comparing and prioritizing the objects under subjective judgments, when quantitative measurements are either not available or accurate assessments are not possible. This technique is also known as special rank order technique. Under this technique, objects/items are presented to judges in the form of pairs and judges are asked to select the best one according to some specific criterion. This process is repeated for several pairs. Recently, this technique is applied in different fields, such as sensory experiments, taste testing, medicine, marketing, business decision-making, investigating preferences, and choice behavior. A huge amount of literature is available on the subject. Abbas and Aslam [1] analyzed the Pareto model under paired comparison techniques by using classical and Bayesian framework. For the
Bayesian analysis they used cricket teams' real dataset. Fitness of the model was judged using Chi square statistic. They found that their suggested model is suitable for the paired comparison data and the prior knowledge affect conclusions. Dittrich et al. [2] discussed a method for the analysis of Likert responses' measurements on attitudinal scale by focusing the relative importance of items in the data. They converted the Likert responses into pairwise comparison responses; then, the multivariate pattern of responses is analyzed. Buck and Wakeling [3] gave the idea of a predictive model to know the consumers preferences for the sensory panel means and relative proportion for the products in pair comparisons. They suggested Binomial discrete logistic model for the analysis. A modification is made to adjust consumers equally liking or disliking. More than 500 consumers' preference datasets were taken for analysis of the model. Abbas and Aslam [4] analyzed various 
factors which generally influence the judges' opinions about the objects using the mixture model for the preference data. Probabilistic features of the mixture distribution besides inferential and computational problems arising out of maximum likelihood estimation are discussed. Dewart and Gillard [5] introduced a new criterion for comparing two ranking schemes using the Bradley and Terry model. Their forecasting and prediction results were based on the outcomes of cricket test matches. The predictions obtained showed better results compared to the prevailing forecasts. They also gave their own rating for the individual player to predict results for the upcoming matches. The purpose of their study was to show the importance and applications of the Bradley and Terry model in the field of paired comparisons. Altaf et al. [6] used noninformative and informative priors for the analysis of the amended Davidson model. The analysis was carried out on the case study. Their results showed that the model under the study is appropriate for qualitative data. In the literature, different PC models exist which are helpful in ranking items. Thurstone [7], Bradley and Terry [8], Stern [9], and Abbas and Aslam [10] constructed different PC models. Ullah et al. [11] suggested the Weibull PC model and analyzed it using Bayesian approach. Annis and Craig [12] and Cattelan [13] used PC technique to analyze sports-related problems. For more details on PC models and numerical approximation, one may see Schauberger and Tutz [14], Dittrich at al. [15], Van et al. [16], Kifyat et al. [17], Sung and Wu [18], Cui et al. [19], Kumar et al. [20], Ahmad et al. [21], Kumar et al. [22], and Ishkhanyan et al. [23].

Our study aims to contribute in the field of Bayesian statistics especially in paired comparisons and analyzing the suggested model under noninformative priors using numerical approximation as it is the leading feature of our study and has not been discussed previously in the literature.

In this study, Section 1 defines material and methods which include development and notations, Bayesian analysis of the Weibull PC model using prior distributions such as (Uniform and Jeffreys) priors, and graphical presentation of the marginal densities of the model's worth parameters. Results and discussions are presented in Section 3. Concluding remarks of the study are provided in Section 4.

\section{Materials and Methods}

2.1. The Weibull Paired Comparison Model. The basics for the development of the Weibull PC model is elaborated in Ullah et al. [11]. According to the Weibull PC model, the probability of preferring treatment $T_{j}$ over treatment $T_{k}$ is denoted by $\theta_{j \cdot j k}$ and can be calculated as follows:

$$
\theta_{j \cdot j k}=\frac{\gamma_{j}^{3}}{\gamma_{j}^{3}+\gamma_{k}^{3}},
$$

and similarly the probability of preferring treatment $T_{k}$ over treatment $T_{j}$ denoted by $\theta_{k \cdot j k}$ is defined below:

$$
\theta_{k \cdot j k}=\frac{\gamma_{k}^{3}}{\gamma_{j}^{3}+\gamma_{k}^{3}} .
$$

Let $r=r_{j k}=r_{k j}$, pairwise comparisons of two treatments $\left(T_{j}, T_{k}\right)$. If $b_{j k}$ denotes the preferences for treatment $T_{j}$ to treatment $T_{k}$ with $b_{k j}=r-b_{j k}$, i.e., $r=b_{j k}+b_{k j}$, then the likelihood function for the results obtained in the trial " $b$ " with data $\left(b_{j k}, b_{k j}\right)$ and the parameters $\gamma=\gamma_{1}, \gamma_{2}, \ldots, \gamma_{h}$ is

$$
l(b, \gamma) \propto \prod_{j<k=1}^{h} \frac{\gamma_{j}^{3 b_{j k}} \gamma_{k}^{3 b_{k j}}}{\left(\gamma_{j}^{3}+\gamma_{k}^{3}\right)^{b_{j k}+b_{j k}} .}
$$

We impose a constraint on the model's parameters, i.e., $\sum_{j=1}^{h} \gamma_{j}=1$. Our purpose of imposing this condition for the confirmation of well-defined parameters.

2.2. Prior Distributions for Parameters of the Weibull PC Model. For the Bayesian analysis, two noninformative (Uniform and Jeffreys) priors are assumed. The noninformative uniform prior allocates identical probability to each and every unit. Symbolically, we can write it as follows:

$$
p_{u}(\gamma) \propto 1,
$$

while the Jeffreys prior can be obtained as follows:

$$
p_{J}(\gamma) \propto \sqrt{\operatorname{det}\{I(\gamma)\}} .
$$

For $m=2$, we have fisher's information matrix is as follows:

$$
I(\gamma)=(-1)^{2}\left|\begin{array}{ll}
E\left\{\frac{\partial^{2} \log l(\cdot)}{\partial \gamma_{1}^{2}}\right\} & E\left\{\frac{\partial^{2} \log l(\cdot)}{\partial \gamma_{1} \partial \gamma_{2}}\right\} \\
E\left\{\frac{\partial^{2} \log l(\cdot)}{\partial \gamma_{2} \partial \gamma_{1}}\right\} & E\left\{\frac{\partial^{2} \log l(\cdot)}{\partial \gamma_{2}^{2}}\right\}
\end{array}\right| .
$$

The simplified algebraic expression of the Jeffreys prior for $m=2$ is given below:

$$
p_{J}(\gamma) \propto \sqrt{\frac{\gamma_{1} \cdot \gamma_{2}\left(\gamma_{1}^{6}+9 \gamma_{1}^{3} \gamma_{2}^{3}-\gamma_{2}^{6}\right)}{\left(\gamma_{1}^{3}+\gamma_{2}^{3}\right)^{4}}} .
$$

A program was designed in Mathematica 8.0 to solve the Jeffreys prior algebraically. As the Jeffreys prior have long and complicated algebraic expression which is not easy for applying for $m=5$, so we drive the Jeffreys prior numerically for $m=5$, designed in SAS package and can be obtain from the first author upon the request.

2.2.1. Joint and Marginal Posterior Distributions of the Weibull PC Model. The joint posterior distribution of the Weibull model parameters given in (8) with prior distribution $p(\gamma)$ is 


$$
P\left(\gamma_{1}, \ldots, \gamma_{h} \mid b\right) \propto p(\gamma) \prod_{j<k=1}^{h} \frac{\gamma_{j}^{3 b_{j k}} \gamma_{k}^{3 b_{k j}}}{\left(\gamma_{j}^{3}+\gamma_{k}^{3}\right)^{b_{j k}+b_{j k}}}
$$

The marginal posterior distribution of Weibull paired comparison model parameter $\gamma_{1}$ given data using uniform and Jeffreys priors is defined in (9) and (10), respectively:

$$
\begin{aligned}
& p\left(\gamma_{1} \mid b\right)=\frac{1}{C} \int_{0}^{1-\gamma_{1}} \int_{0}^{1-\gamma_{1}-\gamma_{2}} \int_{0}^{1-\gamma_{1}-\gamma_{2}-\gamma_{3}} p_{u}(\gamma) \prod_{j<k=1}^{h} \frac{\gamma_{j}^{3 b_{j k}} \gamma_{k}^{3 b_{k j}}}{\left(\gamma_{j}^{3}+\gamma_{k}^{3}\right)^{b_{j k}+b_{j k}}} \mathrm{~d} \gamma_{4} \mathrm{~d} \gamma_{3} \mathrm{~d} \gamma_{2}, \\
& p\left(\gamma_{1} \mid b\right)=\frac{1}{C} \int_{0}^{1-\gamma_{1}} \int_{0}^{1-\gamma_{1}-\gamma_{2}} \int_{0}^{1-\gamma_{1}-\gamma_{2}-\gamma_{3}} p_{J}(\gamma) \prod_{j<k=1}^{h} \frac{\gamma_{j}^{3 b_{j k}} \gamma_{k}^{3 b_{k j}}}{\left(\gamma_{j}^{3}+\gamma_{k}^{3}\right)^{b_{j k}+b_{j k}}} \mathrm{~d} \gamma_{4} \mathrm{~d} \gamma_{3} \mathrm{~d} \gamma_{2},
\end{aligned}
$$

where $C$ is the normalizing constant and is defined as follows:

$$
C=\int_{0}^{1} \int_{0}^{1-\gamma_{1}} \int_{0}^{1-\gamma_{1}-\gamma_{2}} \int_{0}^{1-\gamma_{1}-\gamma_{2}-\gamma_{3}} p(\gamma) \prod_{j<k=1}^{h} \frac{\gamma_{j}^{3 b_{j k}} \gamma_{k}^{3 b_{k j}}}{\left(\gamma_{j}^{3}+\gamma_{k}^{3}\right)^{b_{j k}+b_{j k}}} \mathrm{~d} \gamma_{4} \mathrm{~d} \gamma_{3} \mathrm{~d} \gamma_{2} \mathrm{~d} \gamma_{1}
$$

Quadrature method is used to evaluate the expression given in (9) and (10). Similarly, the remaining marginal posterior distributions can be obtained numerically.

The analysis of the model dataset given in Table 1 has been collected from the 30 people living in Islamabad, Pakistan, showing their usage preference for the cellphone brands (Huawei (HW), Samsung (SS), Oppo (OP), QMobile (QM), and Nokia (NK)).

2.3. Graphical Analysis of the Marginal Posterior Distributions. The graphical representation of the marginal densities of the model's worth parameters $\gamma_{1}, \gamma_{2}, \gamma_{3}, \gamma_{4}$, and $\gamma_{5}$ using Uniform and Jeffreys priors for the Weibull PC model are shown in Figures 1 and 2, respectively.

Glancing through the above figures, we observe that all the graphs of the marginal posterior distributions are approximately symmetrical. Section 2 explained the model development, model notations, and graphical analysis under both of the priors. In Section 3, results are obtained using the quadrature method.

\section{Results and Discussion}

3.1. Posterior Estimates and Posterior Risks. Loss function indicates the difference between the parameter and estimate. It is used for the estimation of the parameter. It describes the accuracy of the method used to analyze the dataset. When the loss function is used, the Bayesian estimator minimizes the expected loss with respect to the posterior distribution. The term Bayes estimator (BE) can be also regarded as Bayes action. It minimizes posterior expected value of the loss function. The expected value calculated is considered as Bayes estimate (BE), while the expected loss is represented by the Posterior Risk (PR). Larger value of the posterior risk is the indication of higher posterior risk, while the smaller value is the sign of higher reliability. This section presents the derivation of the Bayes Estimators and Posterior Risks (PRs) under loss functions using Uniform and Jeffreys priors. We used four different loss functions named as Squared Error Loss Function (SELF), Quadratic Loss Function (QLF), DeGroot Loss Function (DLF), and Precautionary Loss Function (PLF) [11]. The BEs and PRs, using various loss functions under Uniform and Jeffreys priors, are obtained and given in Tables 2 and 3. Results show that PRs for the parameters of $\gamma_{j}, j=1,2, \ldots, 5$ under SELF are smaller when compared to the remaining loss functions: DLF, PLF, and QLF. Thus, the results clearly show that SELF is the most suitable loss function for the estimation of model's parameters $\gamma_{1}, \gamma_{2}, \gamma_{3}, \gamma_{4}$, and $\gamma_{5}$. From the posterior estimates, it becomes now clear that the ranking order for the cellphone brand usage preference is as follows: $\mathrm{SS} \longrightarrow \mathrm{HW} \longrightarrow \mathrm{OP} \longrightarrow \mathrm{NK} \longrightarrow \mathrm{QM}$.

3.2. Preference Probabilities. The probabilities that show the chance of preferring any cellphone brand $T_{j}$ over the other cellphone brand $T_{k}$ are known as preference probabilities. The posterior estimates are used to calculate preference probabilities. The preference probabilities for the worth parameters $\gamma_{j}, j=1,2,3,4,5$, are obtained using posterior estimates of uniform prior under SELF through numerical approximation. The preference probabilities calculated are displayed in Table 4.

The value for the Huawei and Samsung cellphone brands' pair (HW, SS) indicates that Huawei has probability 0.4928 of being preferred over the Samsung, and the probability $(0.5072)$ is in the favor of Samsung against the 
TABLE 1: Data regarding the cellphone brand usage preferences.

\begin{tabular}{lccccccccccc}
\hline Pairs $(\mathrm{j}, \mathrm{k})$ & $(1,2)$ & $(1,3)$ & $(1,4)$ & $(1,5)$ & $(2,3)$ & $(2,4)$ & $(2,5)$ & $(3,4)$ & $(3,5)$ & $(4,5)$ \\
\hline Pairs of cellphones & $(\mathrm{HW}$, & $(\mathrm{HW}$, & $(\mathrm{HW}$, & $(\mathrm{HW}$, & $(\mathrm{SS}$, & $(\mathrm{SS}$, & $(\mathrm{SS}$, & $(\mathrm{OP}$, & $(\mathrm{OP}$, & $(\mathrm{QM}$, \\
brands & $\mathrm{SS})$ & $\mathrm{OP})$ & $\mathrm{QM})$ & $\mathrm{NK})$ & $\mathrm{OP})$ & $\mathrm{QM})$ & $\mathrm{NK})$ & $\mathrm{QM})$ & $\mathrm{NK})$ & $\mathrm{NK})$ \\
$b_{j k}$ & 17 & 16 & 19 & 21 & 22 & 21 & 18 & 20 & 21 & 11 \\
$b_{k j}$ & 13 & 14 & 11 & 9 & 8 & 9 & 12 & 10 & 9 & 19 \\
$n$ & 30 & 30 & 30 & 30 & 30 & 30 & 30 & 30 & 30 & 30 \\
\hline
\end{tabular}

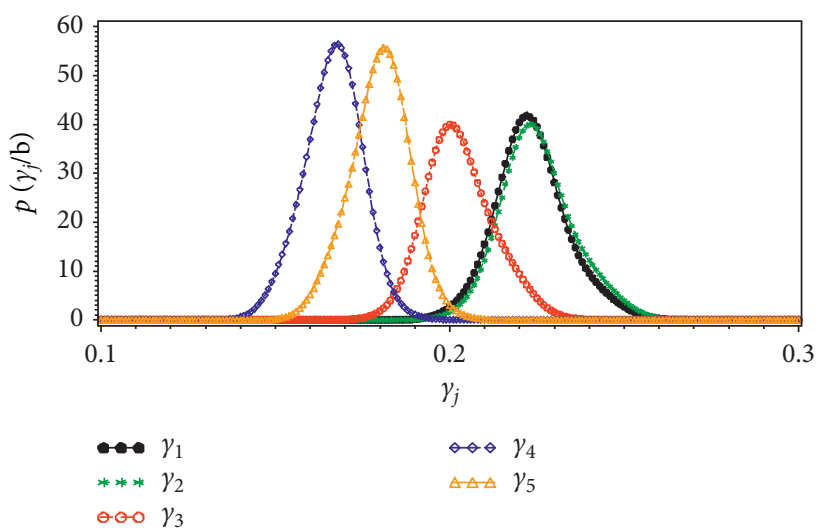

FIgURE 1: The marginal posterior distributions using uniform prior.

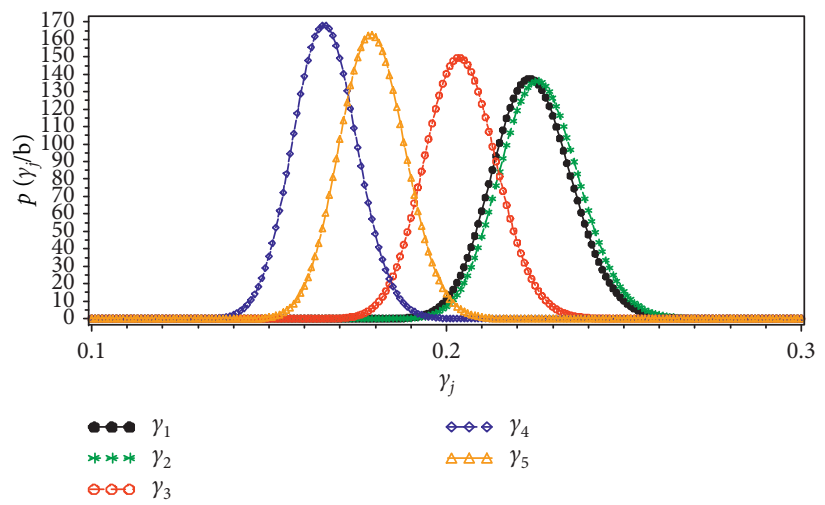

FIgURE 2: The marginal posterior distributions using Jeffreys prior.

Huawei. The remaining probabilities can be interpreted on the same lines. From the results, we observe that there exists a complete coordination in the ranking order with respect to the posterior means and preference probabilities in the both noninformative priors.

3.3. Predictive Probabilities. The predictive probability $\mathrm{P}(j k)$ indicates the future preference of cellphone brand $T_{j}$ over the cellphone brand $T_{k}$ in a single future comparison. The predictive probabilities given in Table 5 are calculated under noninformative (Uniform and Jeffreys) priors using numerical approximation.

The predictive probability 0.4929 predicts that there are $49.29 \%$ chances that the HW will be preferred to SS and $50.71 \%$ chances that SS is preferred to HW in a single future comparison. The remaining predictive probabilities can be interpreted on the same lines. From the results, we observe that the predictive probabilities portray the same ranking order as shown by the posterior means.

3.4. Bayesian Hypothesis Testing. Bayesian hypothesis testing is a simple and straightforward procedure. The posterior probabilities are calculated, and decision between the hypotheses is directly made. We consider the following two hypotheses for comparing $\gamma_{j}$ and $\gamma_{k}$ of any two cellphone brands:

$$
\begin{aligned}
& H_{j k}: \gamma_{j} \geq \gamma_{k} \text { against, } \\
& \bar{H}_{j k}: \gamma_{j}<\gamma_{k} .
\end{aligned}
$$

The posterior probabilities for $H_{j k}$ and $\bar{H}_{k j}$ can be calculated as 
TABLE 2: Bayes estimates (BEs) and posterior risks (PRs) using uniform prior.

\begin{tabular}{|c|c|c|c|c|c|}
\hline \multirow{2}{*}{ Loss functions } & \multicolumn{5}{|c|}{ Parameters } \\
\hline & $\gamma_{1}$ & $\gamma_{2}$ & $\gamma_{3}$ & $\gamma_{4}$ & $\gamma_{5}$ \\
\hline$\overline{\text { SELF }}$ & $0.2240(0.0001)$ & $0.2346(0.0002)$ & $0.2041(0.0001)$ & $0.1658(0.0001)$ & $0.1715(0.0008)$ \\
\hline PLF & $0.2245(0.0005)$ & $0.2348(0.0006)$ & $0.2042(0.0050)$ & $0.1661(0.0004)$ & $0.1704(0.0005)$ \\
\hline QLF & $0.2231(0.0024)$ & $0.2345(0.0025)$ & $0.2032(0.0025)$ & $0.1648(0.0030)$ & $0.1744(0.0027)$ \\
\hline DLF & $0.2248(0.0024)$ & $0.2315(0.0025)$ & $0.2047(0.0025)$ & $0.1645(0.0030)$ & $0.1745(0.0027)$ \\
\hline
\end{tabular}

TABLE 3: Bayes estimates (BEs) and posterior risks (PRs) using Jeffreys prior.

\begin{tabular}{|c|c|c|c|c|c|}
\hline \multirow{2}{*}{ Loss functions } & \multicolumn{5}{|c|}{ Parameters } \\
\hline & $\gamma_{1}$ & $\gamma_{2}$ & $\gamma_{3}$ & $\gamma_{4}$ & $\gamma_{5}$ \\
\hline SELF & $0.2253(0.0001)$ & $0.2346(0.0002)$ & $0.2060(0.0003)$ & $0.1625(0.0005)$ & $0.1716(0.0007)$ \\
\hline PLF & $0.2256(0.0005)$ & $0.2354(0.0006)$ & $0.2050(0.0004)$ & $0.1638(0.0007)$ & $0.1701(0.0009)$ \\
\hline QLF & $0.2242(0.0023)$ & $0.2356(0.0024)$ & $0.2063(0.0022)$ & $0.1628(0.0029)$ & $0.1711(0.0027)$ \\
\hline DLF & $0.2258(0.0024)$ & $0.2345(0.0023)$ & $0.2065(0.0026)$ & $0.1630(0.0029)$ & $0.1702(0.0026)$ \\
\hline
\end{tabular}

TABLE 4: The preference probabilities using uniform prior.

\begin{tabular}{lcccccccccc}
\hline Pairs & $(\mathrm{HW}, \mathrm{SS})$ & $(\mathrm{HW}, \mathrm{OP})$ & $(\mathrm{HW}, \mathrm{QM})$ & $(\mathrm{HW}, \mathrm{NK})$ & $(\mathrm{SS}, \mathrm{OP})$ & $(\mathrm{SS}, \mathrm{QM})$ & $(\mathrm{SS}, \mathrm{NK})$ & $(\mathrm{OP}, \mathrm{QM})$ & $(\mathrm{OP}, \mathrm{NK})$ & $(\mathrm{QM}, \mathrm{NK})$ \\
\hline$P_{j . j k}$ & 0.4928 & 0.5699 & 0.7120 & 0.6623 & 0.5770 & 0.7179 & 0.6687 & 0.6512 & 0.5967 & 0.4422 \\
$P_{k . j k}$ & 0.5072 & 0.4301 & 0.2880 & 0.3377 & 0.4230 & 0.2821 & 0.3313 & 0.3488 & 0.4033 & 0.5578 \\
$P_{j . j k}$ & 0.4930 & 0.5663 & 0.7270 & 0.6681 & 0.5732 & 0.7325 & 0.6742 & 0.6709 & 0.6064 & 0.4304 \\
$P_{k . j k}$ & 0.5070 & 0.4337 & 0.2730 & 0.3319 & 0.4268 & 0.2675 & 0.3258 & 0.3291 & 0.3936 & 0.5696 \\
\hline
\end{tabular}

TABle 5: Predictive probabilities using uniform prior.

\begin{tabular}{lcccccccccc}
\hline Pairs & $(\mathrm{HW}, \mathrm{SS})$ & $(\mathrm{HW}, \mathrm{OP})$ & $(\mathrm{HW}, \mathrm{QM})$ & $(\mathrm{HW}, \mathrm{NK})$ & $(\mathrm{SS}, \mathrm{OP})$ & $(\mathrm{SS}, \mathrm{QM})$ & $(\mathrm{SS}, \mathrm{NK})$ & $(\mathrm{OP}, \mathrm{QM})$ & $(\mathrm{OP}, \mathrm{NK})$ & $(\mathrm{QM}, \mathrm{NK})$ \\
\hline $\mathrm{P}_{(j k)}$ & 0.4929 & 0.5690 & 0.7096 & 0.6603 & 0.5759 & 0.7154 & 0.6666 & 0.6492 & 0.5955 & 0.4429 \\
$\mathrm{P}_{(k j)}$ & 0.5071 & 0.4310 & 0.2904 & 0.3397 & 0.4241 & 0.2846 & 0.3334 & 0.3508 & 0.4045 & 0.5571 \\
$\mathrm{P}_{(j k)}$ & 0.4931 & 0.5655 & 0.7245 & 0.6666 & 0.5722 & 0.7300 & 0.6721 & 0.6691 & 0.6052 & 0.4312 \\
$\mathrm{P}_{(k j)}$ & 0.5069 & 0.4345 & 0.2755 & 0.3334 & 0.4278 & 0.2700 & 0.3279 & 0.3309 & 0.3948 & 0.5688 \\
\hline
\end{tabular}

TABLE 6: Posterior probabilities using uniform prior.

\begin{tabular}{lcccccccccc}
\hline Pairs & $p_{12}$ & $p_{13}$ & $p_{14}$ & $p_{15}$ & $p_{23}$ & $p_{24}$ & $p_{25}$ & $p_{34}$ & $p_{35}$ & $p_{45}$ \\
\hline$H_{j k}$ & 0.3432 & 0.8165 & 0.9997 & 0.9946 & 0.8473 & 0.9998 & 0.9962 & 0.9895 & 0.9107 & 0.0877 \\
$H_{k j}$ & 0.6568 & 0.1835 & 0.0003 & 0.0054 & 0.1527 & 0.0002 & 0.0038 & 0.0105 & 0.0893 & 0.9123 \\
\multicolumn{7}{c}{} & & \multicolumn{7}{c}{ Posterior probabilities using Jeffreys prior } & & \\
$H_{j k}$ & 0.3429 & 0.8073 & 0.9998 & 0.9964 & 0.8388 & 0.9999 & 0.9974 & 0.9952 & 0.9408 & 0.0600 \\
$H_{k j}$ & 0.6571 & 0.1927 & 0.0002 & 0.0036 & 0.1612 & 0.0001 & 0.0026 & 0.0048 & 0.0592 & 0.9400 \\
\hline
\end{tabular}

$$
p_{j k}=p\left(H_{j k}\right)=\int_{\delta=0}^{1} \int_{\varphi=\delta}^{((1+\delta) / 2)} p(\delta, \varphi \mid a) \mathrm{d} \varphi \mathrm{d} \delta
$$

where $\delta=\gamma_{j}-\gamma_{k}, \varphi=\gamma_{j}$, and $q_{j k}=p\left(\bar{H}_{j k}\right)=1-p\left(H_{j k}\right)$

The hypothesis with higher probability will be accepted. The posterior probabilities for $H_{j k}$ and $\bar{H}_{j k}$ are computed using numerical approximation and given in Table 6 under both (Uniform and Jeffreys) priors.

From the results we can conclude that the hypotheses $H_{12}$ and $H_{45}$ are rejected and $H_{13}$ and $H_{23}$ are inconclusive, while the remaining are accepted indicating SS is preferred over HW, NK, OP, and QM, respectively. Similarly, HW is preferred over SS, NK, OP, and QM, respectively. We can also interpret the remaining posterior probabilities on the same lines.

3.5. Plausibility of the Model. Chi Square test is used to check the plausibility of the model under study. Model is said to be plausible if the calculated and expected frequencies are closer to the corresponding observed frequencies. Let $b_{j k}$ and $b_{k j}$ denote the expected frequencies, which can be calculated as $\hat{b}_{j k}=r\left(\gamma_{j}^{3} /\left(\gamma_{j}^{3}+\gamma_{k}^{3}\right)\right)$ and 
$\bar{b}_{k j}=r\left(\gamma_{k}^{3} /\left(\gamma_{j}^{3}+\gamma_{k}^{3}\right)\right)$. The calculated value of the Chi Square statistic for the Weibull PC model is 4.64 with $p$ value 0.59075 at 6 degrees of freedom. So, according to the decision rule, it is quite evident that the model under study is plausible to the data. Similar results are obtained using the Jeffreys prior.

\section{Conclusion}

The Weibull PC model is analyzed under Bayesian paradigm using noninformative (Uniform and Jeffreys) priors through numerical approximation. The dataset for the usage preferences of the cellphone brands is collected from the people living in Islamabad, Pakistan, and used for the Bayesian analysis. The posterior distributions are derived and the posterior estimates are obtained under various loss functions such as Squared Error Loss Function, Quadratic Loss Function, DeGroot Loss Function, and Precautionary Loss Function. The graphical representation of the marginal posterior distributions of the model parameters has revealed the symmetry of the distributions of the parameters. It has been observed from the Bayes estimates using both priors that the usage preference for the cellphone brands under study may be ranked as $\mathrm{SS} \longrightarrow \mathrm{HW} \longrightarrow \mathrm{OP} \longrightarrow \mathrm{NK} \longrightarrow \mathrm{QM}$. The preference probabilities of current pairwise comparison and the predictive probabilities of future pairwise comparison have been presented in the Bayesian analysis. The posterior probabilities of hypotheses to compare two parameters are discussed in this study. Moreover, the model has been proved to be plausible in the light of observed data as it yields a Chi square value of 4.64 with an associated $p$ value of 0.59075 . We suggest that this model may be treated as a good candidate to rank any types of competitors besides those studied here.

\section{Data Availability}

The data for the research work are personally collected from the people living in capital city of Pakistan.

\section{Conflicts of Interest}

The authors declare that there are no conflicts of interest regarding the publication of this article.

\section{References}

[1] N. Abbas and M. A. Aslam, "Bayesian modeling to paired comparison data via the Pareto distribution," Pakistan Journal of Statistics and Operation Research, vol. 13, no. 4, pp. 875-891, 2017.

[2] R. Dittrich, B. Francis, R. Hatzinger, and W. Katzenbeisser, "A paired comparison approach for the analysis of sets of likertscale responses," Statistical Modelling: An International Journal, vol. 7, no. 1, pp. 3-28, 2007.

[3] D. Buck and I. Wakeling, "Predicting paired preferences from sensory data," Food Quality and Preference, vol. 12, pp. 481-487, 2001.

[4] N. Abbas and M. Aslam, "Extending the Bradley-Terry model for paired comparisons to accommodate weights," Journal of Applied Statistics, vol. 38, no. 3, pp. 571-580, 2011.
[5] N. Dewart and J. Gillard, "Using bradley-terry model to analyze test match cricket," IMA Journal of Management Mathematics, vol. 30, no. 2, pp. 187-207, 2019.

[6] S. Altaf, M. Aslam, and M. Liaqat, "Decision-making for paired comparison using the extended amended Davidson model," Communications in Statistics-Theory and Methods, vol. 46, no. 4, pp. 1766-1778, 2017.

[7] L. L. Thurstone, "A law of comparative judgment," Psychological Review, vol. 34, no. 4, pp. 273-286, 1927.

[8] R. A. Bradley and M. E. Terry, "Rank analysis of incomplete block designs," Biometrika, vol. 39, no. 3-4, pp. 324-345, 1952.

[9] H. Stern, "A continuum of paired comparisons models," Biometrika, vol. 77, no. 2, pp. 265-273, 1990.

[10] N. Abbas and M. Aslam, "Prioritizing the items through paired comparison models, A bayesian approach," Pakistan Journal of Statistics, vol. 25, no. 1, pp. 59-69, 2009.

[11] K. Ullah, M. Aslam, and T. N. Sindhu, "Bayesian analysis of the Weibull paired comparison model using informative prior," Alexandria Engineering Journal, vol. 59, no. 4, pp. 2371-2378, 2020.

[12] D. H. Annis and B. A. Craig, "Hybrid paired comparison analysis, with applications to the ranking of college football teams," Journal of Quantitative Analysis in Sports, vol. 1, no. 1, pp. 1-31, 2005.

[13] M. Cattelan, "Models for paired comparison data: a review with emphasis on dependent data," Statistical Science, vol. 27, no. 3, pp. 412-433, 2012.

[14] G. Schauberger and G. Tutz, "Subject-specific modelling of paired comparison data: a lasso-type penalty approach," Statistical Modelling: An International Journal, vol. 17, no. 3, pp. 223-243, 2017.

[15] R. Dittrich, B. Francis, R. Hatzinger, and W. Katzenbeisser, "Missing observations in paired comparison data," Statistical Modelling, vol. 12, no. 2, pp. 117-143, 2012.

[16] W. van, P. Solymos, K. Kardynal, and M. Frey, "Paired sampling standardizes point count data from humans and acoustic recorders," Avian Conservation and Ecology, vol. 12, no. 1, pp. 1-15, 2017.

[17] T. Kafyat, M. Aslam, and S. A Cheema, "The maxwell paired comparison model under bayesian paradigm using informative priors," Communication in Statistics-Theory and Methods, 2020

[18] Y.-T. Sung and J.-S. Wu, "The visual analogue scale for rating, ranking and paired-comparison (VAS-RRP): a new technique for psychological measurement," Behavior Research Methods, vol. 50, no. 4, pp. 1694-1715, 2018.

[19] W. Cui, X.-Y. Peng, and Z.-Z. Yan, "Bayesian analysis of a constant-stress accelerated life testing with thermal aging life model under general progressive type-II censored data," Thermal Science, vol. 23, no. 4, pp. 2509-2516, 2019.

[20] S. Kumar, D. Kumar, J. R. Sharma, C. Cesarano, P. Agarwal, and C. Y. Ming, "An optimal fourth order derivative-free numerical algorithm for multiple roots," Symmetry, vol. 12, pp. 1-14, 2020.

[21] I. Ahmad, H. Ahmad, T. Phatiphat, Y. M. Chu, and C. Cesarano, "Solution of multi-term time-fractional PDE models arising in mathematical biology and physics by local meshless method," Symmetry, vol. 12, pp. 1-11, 2020.

[22] D. Kumar, J. R. Sharma, and C. Cesarano, "An efficient class of traub-steffensen-type methods for computing multiple zeros," AXIOMS, vol. 8, pp. 1-11, 2019.

[23] A. Ishkhanyan and C. Cesarano, "Generalized-hypergeometric solutions of the general fuchsian linear ODE having five regular singularities," AXIOMS, vol. 8, pp. 1-8, 2019. 